\title{
Variations in the Flow Approach to CFCLP-TC for Multiobjective Supply Chain Design
}

\author{
Minor P. Hertwin, ${ }^{1}$ Olivares-Benítez Elías, ${ }^{2}$ \\ Tapia-Olvera Ruben, ${ }^{1}$ and Martínez Flores J. Luis ${ }^{2}$ \\ ${ }^{1}$ School of Engineering, Polytechnic University of Tulancingo, Tulancingo 43629, Mexico \\ ${ }^{2}$ School of Engineering, UPAEP University, Puebla 72410, Mexico \\ Correspondence should be addressed to Minor P. Hertwin; hertwin.minor@upt.edu.mx
}

Received 18 October 2013; Accepted 25 December 2013; Published 13 March 2014

Academic Editor: Timothy I. Matis

Copyright (C) 2014 Minor P. Hertwin et al. This is an open access article distributed under the Creative Commons Attribution License, which permits unrestricted use, distribution, and reproduction in any medium, provided the original work is properly cited.

\begin{abstract}
We review the problem for the design of supply chains called Capacitated Fixed Cost Facility Location Problem with Transportation Choices (CFCLP-TC). The problem is based on a production network of two echelons with multiple plants, a set of potential distribution centers, and customers. The problem is formulated as an optimization model with two objective functions based on time and cost. This paper proposes three changes to the original model to compare the sets of efficient solutions and the computational time required to obtain them. The main contribution of this paper is to extend the existing literature by incorporating approaches for the supply of product to customers through multiple sources, the direct flow between plants and customers, without this necessarily implying removing the distribution centers, and the product flow between distribution centers. From these approaches, we generate mathematical programming models and propose to solve through the epsilon-constraint approach for generating Pareto fronts and thus compare each of these approaches with the original model. The models are implemented in GAMS and solved with CPLEX.
\end{abstract}

\section{Introduction}

Supply Chain Management (SCM) is the process of planning, implementing, and controlling the operation of the supply chain efficiently. SCM spans all movements and storage of raw materials, work-in-process inventory, and finished goods from the point of origin to the point of consumption [1]. Part of the planning processes in SCM aim at finding the best possible supply chain configuration so that all operations can be performed in an efficient way. The Capacitated Fixed Cost Facility Location Problem with Transportation Choices (CFCLP-TC) proposed by $[2,3]$ is a combinatorial optimization problem for supply chain design. It is an extension of the CFLP as a biobjective mixed-integer program. It is based on a two-echelon system for the distribution of one product in a single time period with two objectives: to minimize cost and to minimize the time of transportation from plants to customers. This approach considers several alternatives to transport the product from one facility to the other in each echelon of the network. The criterion of cost is an aggregate function of variable cost and fixed cost. At difference from similar works in the literature, the aim here is to provide the decision maker with a set of nondominated alternatives to allow her deciding. Some qualitative information only known by the decision maker may motivate the selection of one of those alternatives.

This paper presents three innovative approaches for the design of a supply chain for biobjective problems. In the first approach, it is proposed that customers can be supplied by more than one distribution center; in the second approach it is proposed that customers can be supplied directly by plants, but the distribution centers can be used. In the third approach proposed here a product flow may exist between the distribution centers. For the study of this problem and the proposed variations, instances of different sizes were used. The results are compared on the basis of three metrics. The first metric is called $R_{\text {pos }}$, proposed by [4], and the second and third metrics are called $D_{\text {avg }}$ and 
$D_{\text {min }}$, proposed by [3] to compare two biobjective Pareto fronts.

This paper is divided into five sections as follows: the first section presents a general introduction to the work. The second section presents the literature review to define the opportunity area that the work will fill. The third section presents an overview of the problem and its variations. The fourth section describes the computational experiment, the metrics used to evaluate the Pareto fronts, and the results of the computational implementation. Finally, in the fifth section the conclusions and proposals to carry out further work are exposed.

\section{Literature Review}

Historically, researchers have focused on the design of distribution systems [5], without considering the supply chain as a whole. Typically, discrete location models were proposed to include additional features. Reference [6] reviews some important mixed-integer formulations for productionsdistribution systems. However, those models had limited scope and could not cope with realistic supply chain structures. Reference [5] proposes the inclusion of relevant features for the Supply Chain Management (SCM) in the facility location models that gradually began to be considered. These include

(a) subsets of the products (customer specifications),

(b) upper and lower limits on shipments of a product to a particular plant,

(c) specifications of the product weights for performance measures in the distribution centers,

(d) piecewise linear approximation for nonlinear costs,

(e) the ability to locate plants and distribution centers,

(f) inclusion of capacity constraints of the products in plants,

(g) conversion of raw materials in activities of one or two levels,

(h) additional distribution and production levels.

Reference [7] suggested the inclusion of additional elements in facility location models such as the inclusion of new objectives (maximum return of investment) and decisions regarding the selection of equipment for new installations. In discrete location problems, selecting sites to establish new facilities in is restricted to a finite set of places available for the location. The simplest example of this approach is where $p$ facilities must be selected to minimize the total distance (weighted) or the costs to supply customer demand.

This is a classic problem called the $p$-median problem, which has been extensively studied by [8-11]. This problem assumes that all candidate sites are equivalent in terms of installation cost for a new facility. When this is not the case, the objective function is extended with a term for the fixed cost of location, and as a result, the number of facilities to be open is an endogenous decision. This new approach is known in the literature as the uncapacitated facility location problem
(UFLP). There are many references to this problem like in [12-14]. In both cases, the $p$-median and UFLP, each client is assigned to an open facility that minimizes the allocation cost. One of the most important extensions to the UFLP is the capacitated facility location problem (CFLP) in which exogenous values are considered to maximize the demand that can be supplied for each potential site. In this case, the closest assignment property is no longer valid as proposed by [15-17].

The models above have several common characteristics as follows:

(a) single period of planning horizon,

(b) deterministic parameters (demand, costs),

(c) single product,

(d) a single type of facility,

(e) decisions of location-allocation.

Clearly, these models are insufficient to handle a realistic facility location scenario. Therefore, many extensions of this basic problem have been proposed and widely studied [18]. A crucial issue in many practical problems of localization is to consider the existence of different types of facilities [19], each playing a specific role (production or storage), and a natural flow of the material (i.e., a hierarchy) between them [20]. Each set of facilities of the same type is usually denoted by a level or echelon in the hierarchy of facilities.

Part of the planning processes in SCM aim at finding the best possible supply chain configuration so that all operations can be performed in an efficient way. The coming back to the capacitated facility location problem (CFLP) is a wellknown combinatorial optimization problem. It consists in deciding which facilities to open from a given potential set, and how to assign customers to those facilities. The objective is minimizing total fixed and shipping cost. Applications of the CFLP include location and distribution planning, lot sizing in production planning, and telecommunication network design as mentioned by [21]. Numerous heuristics and exact algorithms for the CFLP have been proposed in the literature. Heuristic solution methods as well as approximation algorithms were proposed by [22-24]. Tabu Search methods for the related $p$-median problem and the CFLP with single source were developed $[25,26]$. Exact solution methods based on the Benders decomposition algorithm are considered [27]. Polyhedral results for the CFLP have been obtained by [28]. Reference [29] uses these results in a branch and cut algorithm for the CFLP.

Moreover, several variants of the CFLP have been investigated. Reference [30] formulated a stochastic integer linear programming model for the CFLP with stochastic demands. A branch and cut approach was applied to find the optimal solution of the problem. Reference [31] formulated the mathematical model of the two-echelon single-source CFLP and considered six Lagrangian relaxation based approaches for the solution. In the recent years, many metaheuristic approaches have been applied to combinatorial optimization problems successfully, such as Simulated Annealing (SA), Genetic Algorithms (GAs), Tabu Search (TS), and Ant 
Colony Optimization (ACO). Some recent work in this field includes those presented by [32] in which they use an MAX-MIN ant system approach for the design of a supply chain. Reference [33] presented a memetic algorithm for a multistage supply chain problem. Reference [34] proposes a simulated annealing algorithm for an allocation problem. Reference [35] presents a hybrid approach using an artificial bee algorithm (BA) with mixed integer programming (MIP) applied to a large-scale CFLP; BA is applied for the purpose of solving the location problem, and the MIP is applied for the purpose of finding the optimal mathematical problem.

The biobjective location problems are extensions of classic locations problems. These problems are biobjective median, knapsack, quadratic, covering, unconstrained, location-allocation, hub, hierarchical, competitive, network, and undesirable and semidesirable location problems. Considering capacities in location problems, there are capacitated and uncapacitated problems in the literature. For instance, [36] has considered an uncapacitated facility location problem with two maxisum objectives (net profit and profitability of investment) and modeled it as parametric integer program with fractional and linear objectives. Reference [37] has modeled a supply network as a biobjective uncapacitated facility location problem with minisum and maxisum objectives (cost and coverage). In contrast, [38] developed an extension of the capacitated model to deal with locating maternity facilities with minisum objectives (distance traveled and load imbalance). Reference [39] has used a different bicriteria approach to the single hub location/allocation problem. This approach has two objectives; the first has a minisum form (cost), while the second objective (processing time) has two alternative forms.

The Capacitated Fixed Cost Facility Location Problem with Transportation Choices (CFCLP-TC) proposed by [2] is an extension of the CFLP with a biobjective mixed-integer program approach (cost and time). This approach considers several alternatives to transport the product from one facility to the other in each echelon of the network. At difference from similar works in the literature, the aim here is to provide the decision maker with a set of nondominated alternatives to allow him deciding. Some qualitative information only known by the decision maker may motivate the selection of one of those alternatives.

\section{Problem Description}

The Capacitated Fixed Cost Facility Location Problem with Transportation Choices (CFCLP-TC) proposed by [2] is based on a two-echelon system for the distribution of one product in a single time period. In the first step, the product is sent from manufacturing plants $(i)$ to distribution centers (j).

The second step corresponds to the flow of product from distribution centers $(j)$ to customers $(k)$. In this problem, the number and location of plants $(i)$ and customers $(k)$ are known a priori. This includes a further decision on the selection of channels of transportation between facilities

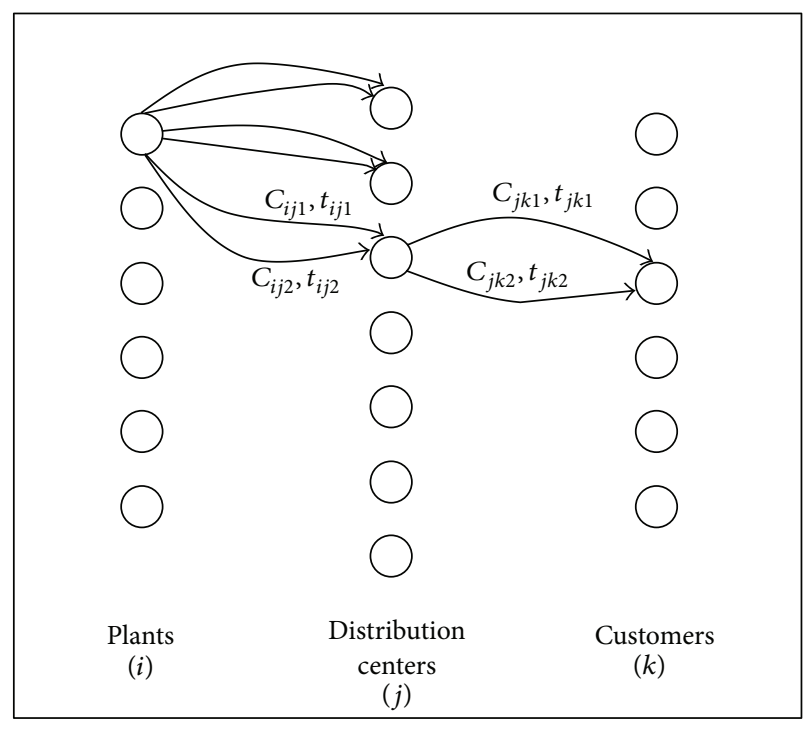

FIgure 1: Schematic of the Capacitated Fixed Cost Facility Location Problem with Transportation Choices (CFCLP-TC).

using a bi-objective approach that simultaneously minimizes the time of transportation of product from plants to customers and the combined costs of locating facilities and transportation. This solution approach builds a set of alternative nondominated solutions for the decision maker. This problem has a set of possible locations for the opening of distribution centers $(j)$ and their number is not defined. Each candidate site has a fixed cost for opening a facility, and each site has limited capacity. Manufacturing plants have limited capacity and their production is sent from each plant to the distribution centers.

An important feature of the problem is to consider various alternatives for transportation of product from one facility to another in each step of the network. Each option represents a type of service with associated costs and time parameters. The existence of third-party logistics companies (3PL) causes that different transportation services are available in the market. The alternatives are generated by the supply of different companies, the availability of different types of services (urgent or regular), and the use of different modes of transportation (truck, train, plane, ship, or intermodal). Commonly, these differences involve an inverse correlation between time and cost; a faster service is more expensive. The outline of the distribution network is displayed in Figure 1.

3.1. Approach That Allows Supplying from Multiple Distribution Centers. In the original model it is established as a restriction that each customer is served by a single source (distribution center $(j)$ ). At this point, we ask what would happen if the delivery to customers from multiple sources is allowed? The main idea of this variation is to allow customers to be supplied in some cases by more than one source (distribution center $(j)$ ). 
The implementation of this variation to the original model will be evaluated over the objective functions (1) and (2) to determine which has a better Pareto front. Also we study the behavior of the time required to obtain results compared with that required in the original model.

Sets

\section{I: Set of plants $i$}

$J$ : Set of potential distribution centers $j$

$K$ : Set of customers $k$

$L P_{i j}$ : Set of $\operatorname{arcs} l$ between nodes $i$ and $j ; i \in I, j \in J$

$L W_{j k}$ : Set of $\operatorname{arcs} l$ between nodes $j$ and $k: j \in J, k \in K$.

\section{Parameters}

$C P_{i j l}$ : Cost of transporting one unit of product from plant $i$ to distribution center $j$ using the arc $l ; i \in I, j \in J, l \in$ $L P_{i j}$

$C W_{j k l}$ : Cost of transporting one unit of product from distribution center $j$ to customer $k$ using the $\operatorname{arc} l ; j \in J, k \in$ $K, l \in L W_{j k}$

$T P_{i j l}$ : Time for transporting any quantity of product from plant $i$ to the distribution center $j$ using $\operatorname{arc} l ; i \in I, j \in$ $J, l \in L P_{i j}$

$T W_{j k l}$ : Time for transporting any quantity of product from distribution center $j$ to customer $k$ using arc $l ; j \in$ $J, k \in K, l \in L W_{j k}$

$M P_{i}$ : Capacity of plant $i ; i \in I$

$M W_{j}$ : Capacity of distribution center $j ; j \in J$

$D_{k}$ : Demand of customer $k ; k \in K$

$F_{j}$ : Fixed cost for opening distribution center $j ; j \in J$.

\section{Decision Variables}

$X_{i j l}$ : Quantity transported from plan $i$ to distribution center $j$ using arc $l ; i \in I, j \in J, l \in L P_{i j}$

$Y_{j k l}$ : Quantity transported from distribution center $j$ to customer $k$ using arc $l ; j \in J, k \in K, l \in L W_{j k}$

$Z_{j}$ : Binary variable equal to 1 if distribution center $j$ is open and equal to 0 otherwise; $j \in J$

$A_{i j l}$ : Binary variable equal to 1 if $\operatorname{arc} l$ is used to transport product from plant $i$ to distribution center $j$ and equal to 0 otherwise; $i \in I, j \in J, l \in L P_{i j}$

$B_{j k l}$ : Binary variable equal to 1 if $\operatorname{arc} l$ is used to transport product from distribution center $j$ to customer $k$ and equal to 0 otherwise; $j \in J, k \in K, l \in L W_{j k}$.

\section{Auxiliary Variables}

$T$ : A variable that computes the longest time that takes sending product from any plant to any customer

$E_{j}^{1}$ : Longest time in the first echelon of the supply chain for active distribution center $j$; that is, $E_{j}^{1}=$ $\max _{i, l}\left(T P_{i j l} A_{i j l}\right) ; i \in I, j \in J, l \in L P_{i j}$
$E_{j}^{2}$ : Longest time in the second echelon of the supply chain for active distribution center $j$; that is, $E_{j}^{2}=$ $\max _{k, l}\left(T W_{j k l} B_{j k l}\right) ; j \in J, k \in K, l \in L W_{j k}$.

Model 1. Consider $\min \left(f_{1}, f_{2}\right)$

$$
\begin{aligned}
f_{1}= & \sum_{i \in I} \sum_{j \in J} \sum_{l \in L P_{i j}} C P_{i j l} X_{i j l} \\
& +\sum_{j \in J} \sum_{k \in K} \sum_{l \in L W_{j k}} C W_{j k l} Y_{j k l}+\sum_{j \in J} F_{j} Z_{j},
\end{aligned}
$$

$f_{2}=T$,

$T-E_{j}^{1}-E_{j}^{2} \geq 0 \quad \forall j \in J$,

$E_{j}^{1}-T P_{i j l} A_{i j l} \geq 0 \quad \forall i \in I, j \in J, l \in L P_{i j}$,

$E_{j}^{2}-T W_{j k l} B_{j k l} \geq 0 \quad \forall j \in J, k \in K, l \in L W_{j k}$,

$\sum_{j \in J} \sum_{l \in L W_{j k}} Y_{j k l}=D_{k} \quad \forall k \in K$,

$\sum_{j \in J} \sum_{l \in L P_{i j}} X_{i j l}=M P_{i} \quad \forall i \in I$,

$M W_{j} Z_{j}-\sum_{j \in J} \sum_{l \in L W_{j k}} Y_{j k l} \geq 0 \quad \forall j \in J$,

$\sum_{j \in J} \sum_{l \in L P_{i j}} X_{i j l}-\sum_{j \in J} \sum_{l \in L W_{j k}} Y_{j k l}=0 \quad \forall j \in J$,

$\sum_{l \in L P_{i j}} A_{i j l} \leq 1 \quad \forall i \in I, j \in J$,

$\sum_{l \in L W_{j k}} B_{j k l} \leq 1 \quad \forall j \in J, k \in K$,

$X_{i j l}-A_{i j l} \geq 0 \quad \forall i \in I, j \in J, l \in L P_{i j}$,

$Y_{j k l}-B_{j k l} \geq 0 \quad \forall j \in J, k \in K, l \in L W_{j k}$,

$M P_{i} A_{i j l}-X_{i j l} \geq 0 \quad \forall i \in I, j \in J, l \in L P_{i j}$,

$M W_{j} B_{j k l}-Y_{j k l} \geq 0 \quad \forall j \in J, k \in K, l \in L W_{j k}$,

$\sum_{i \in I} \sum_{l \in L P_{i j}} A_{i j l}-Z_{j} \geq 0 \quad \forall j \in J$,

$$
T, E_{j}^{1}, E_{j}^{2}, X_{i j l}, Y_{j k l} \geq 0
$$

$\forall i \in I, \quad j \in J, \quad k \in K, \quad l \in L P_{i j}, \quad l \in L W_{j k}$

$$
Z_{j}, A_{i j l}, B_{j k l} \in\{0,1\}
$$

$\forall i \in I, \quad j \in J, \quad k \in K, \quad l \in L P_{i j}, \quad l \in L W_{j k}$.

In this model, the objective function (1) minimizes the transportation costs and the cost of opening the distribution 


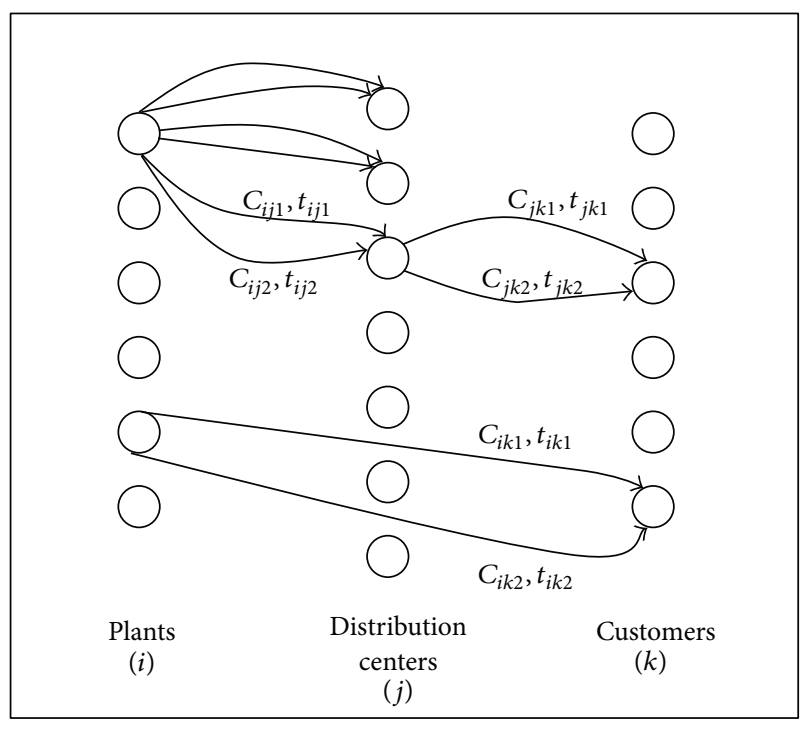

Figure 2: Schematic of the Capacitated Fixed Cost Facility Location Problem with Transportation Choices (CFCLP-TC) with variations in the direct flow between plants $(i)$ and customers $(k)$.

centers. The objective function (2) minimizes the time of transportation. Restriction (3) calculates the longest total time between $(i)$ and $(k)$. The restrictions (4) and (5) allow calculating the transportation time between $(i)$ and $(j)$ and then from $(j)$ to $(k)$. The restriction (6) allows the satisfaction of the demands of each client. The restriction (7) impedes to exceed the capacity of each plant (i). The restriction (8) impedes to exceed the capacity of the distribution centers $(j)$. The restriction (9) allows the balance of flow between $(i)-(j)$ and $(j)-(k)$. Restrictions (10) and (11) provide that the transportation of the material can only be done through a single arc. Restrictions (12) and (13) provide that an arc will be inactive if there is no flow through it. Equations (14) and (15) state the shipment of product only through active arcs. Restriction (16) provides that the distribution centers (j) having no product flow through them must be closed. Restrictions (17) and (18) provide a definition of the variables in the model.

3.2. Approach Allowing the Direct Flow between Plants and Customers. This variation suggests that some plants (i) supply customers in a direct way, that is, without necessarily passing through the distribution centers $(j)$. The main reason is because in some cases customers $(k)$ may be closer to the plants $(i)$ than the distribution centers $(j)$. This could make the flow more efficient in terms of cost and time without considering distribution centers $(j)$. As in the previous variation, we want to evaluate the efficiency of this approach through the construction of a new Pareto front and the performance evaluation of the computational time required. Figure 2 shows the outline of this proposal.

The mathematical model for the variation that allows the direct flow between plants $(i)$ and costumers $(k)$ in some cases is as follows.
Sets

$$
L V_{i k} \text { : Set of } \operatorname{arcs} l \text { between nodes } i \text { and } k: i \in I, k \in K
$$

Parameters

$C V_{i k l}$ : Cost of sending one unit of product from plant $i$ to customer $k$ using the arc $i k l: i \in I, k \in K, l \in L V_{i k}$

$T V_{i k l}$ : Time to transport any quantity of products of plant $i$ to customer $k$ using the arc $i k l ; i \in I, k \in K, l \in L V_{i k}$.

\section{Decision Variables}

$V_{i k l}$ : Quantity transported from plant $i$ to customer $k$ using the arc $i k l ; i \in I, k \in K, l \in L V_{i k}$

$G_{i k l}$ : Binary variable equal to 1 if the arc $i k l$ is used to transport product from plant $i$ to customer $k$ and equals 0 otherwise $i$ and $k ; i \in I, k \in K, l \in L V_{i k}$.

\section{Auxiliary Variables}

E3: The maximum time it takes to ship the $i$ to $k, E_{3}=$ $\max _{k, l}\left(T V_{i k l} G_{i k l}\right) ; i \in I, k \in K, l \in L V_{i k}$.

Model 2. Consider $\min \left(f_{1}, f_{2}\right)$

$$
\begin{aligned}
f_{1}= & \sum_{i \in I} \sum_{j \in J} \sum_{l \in L P_{i j}} C P_{i j l} X_{i j l}+\sum_{j \in J} \sum_{k \in K} \sum_{l \in L W_{j k}} C W_{j k l} Y_{j k l} \\
& +\sum_{i \in I} \sum_{k \in K} \sum_{l \in L V_{i k}} C V_{i k l} V_{i k l}+\sum_{j \in J} F_{j} Z_{j}
\end{aligned}
$$

(2), (3), (4), (5), (8), (9), (11),

(12), (13), (14), (15), (16), (17),

$$
\begin{gathered}
E 3-T V_{i k l} G_{i k l} \geq 0 \quad \forall i \in I, k \in k, l \in L V_{i k} \\
T-E 3 \geq 0 \quad \forall j \in J,
\end{gathered}
$$

$\sum_{j \in J} \sum_{l \in L W_{j k}} Y_{j k l}+\sum_{i \in I} \sum_{l \in L V_{i k}} V_{i k l}=D_{k} \quad \forall i \in I, k \in K$,

$$
\sum_{j \in J} \sum_{l \in L P_{i j}} X_{i j l}+\sum_{i \in I} \sum_{l \in L V_{i k}} V_{i k l}=M P_{i} \quad \forall i \in I, k \in K
$$

$$
\sum_{i \in I} \sum_{l \in L V_{i k}} G_{i k l}+\sum_{j \in J} \sum_{l \in L W_{j k}} B_{j k l}=1 \quad \forall i \in I, k \in K,
$$

$$
\begin{gathered}
\sum_{l \in L V_{i k}} G_{i k l} \leq 1 \quad \forall i \in I, k \in K, \\
V_{i k l}-G_{i k l} \geq 0 \quad \forall i \in I, k \in K, l \in L V_{i k}, \\
M P_{i} G_{i k l}-V_{i k l} \geq 0 \quad \forall i \in I, k \in K, l \in L V_{i k}, \\
T, E_{j}^{1}, E_{j}^{2}, E 3, X_{i j l}, Y_{j k l}, G_{i k l} \geq 0 \\
\forall i \in I, \quad j \in J, \quad k \in K, \\
l \in L P_{i j}, \quad l \in L W_{j k}, \quad l \in L V_{i k},
\end{gathered}
$$




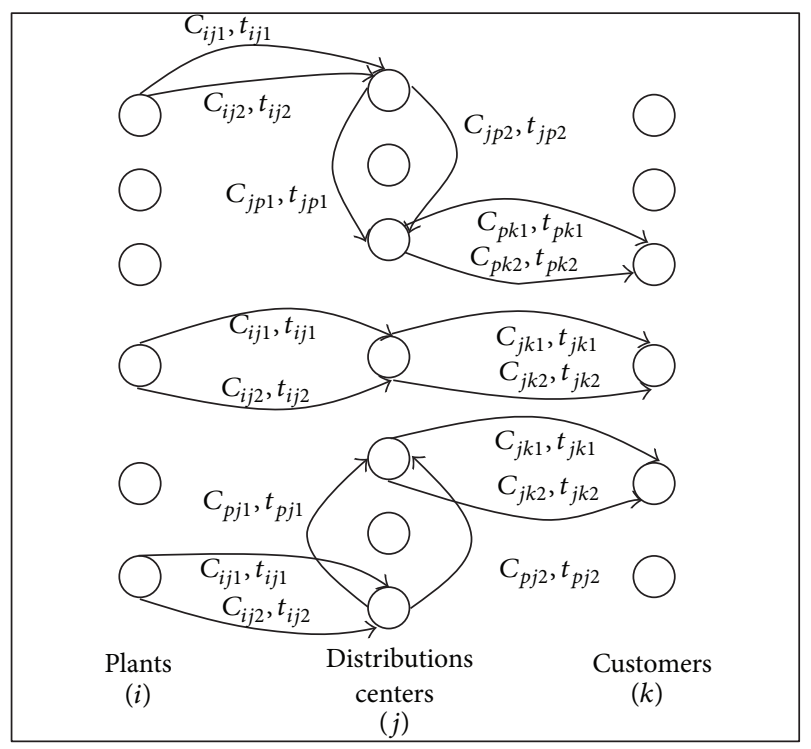

Figure 3: Schematic of the Capacitated Fixed Cost Facility Location Problem with Transportation Choices (CFCLP-TC) flow between distribution centers.

$$
\begin{array}{r}
Z_{j}, A_{i j l}, B_{j k l}, V_{i k l} \in\{0,1\} \\
\forall i \in I, \quad j \in J, \quad k \in K, \quad l \in L P_{i j}, \\
l \in L W_{j k}, \quad l \in L V_{i k} .
\end{array}
$$

In this new formulation (19) replaces (1) in the original formulation as the objective function that searches for the best cost. Equation (20) is added to the original model and this calculates the time between the plants $(i)$ and customers $(k)$. Equation (21) assigns the longest time between plants (i) and customers $(k)$ to variable $T$. Equation (22) replaces (6) in the original model and is aimed at satisfying the customer demand $(k)$. Equation (23) replaces (7) in the original model and this requires that the transported amount from $(i)$ to $(k)$ does not exceed the capacity of the plant $(i)$. Equation (24) is added to the model, and this ensures that customers $(k)$ can be supplied only by a single source. Equation (25) is added to the original model and states that the transportation of the material can only be done through a single arc. Equation (26) is added to the original model to establish that an arc is inactive whether its flow is zero. Equation (27) is added to the original model and states that the product flow will be made only through active arcs. Equations (28) and (29) replace (18) and (19) in the original model and set the domain of the variables.

\subsection{Approach That Allows Flow between Distribution Centers.} This variation suggests the possibility of exchange of goods between distribution centers $(j)$. For some alternatives this variation would compete directly with the flow of plants $(i)$, distribution centers $(j)$, plants $(k)$ with the alternative flow of plants $(i)$, distribution centers $(j)$, distribution centers $(p)$, and plants $(k)$. The main idea is that for some cases it is cheaper and faster to send a product from a distribution center $(j)$ to other distribution centers $(p)$ and then send it to customers $(k)$. Figure 3 shows the outline of this proposal.

The mathematical model allowing flow between distribution centers, $(j)-(p)$, is as follows.

Sets

$L S_{j p}:$ Set of $\operatorname{arcs} l$ between nodes $j-p: j \in J$

$L S_{p j}$ : Set of arcs $l$ between nodes $p-j: j \in J$.

\section{Parameters}

$C S_{j p l}$ : Cost of sending one unit of product between distribution centers $j$ using arc $j p l: j \in J, l \in L S_{j p}$

$C S_{p j l}$ : Cost of sending one unit of product between distribution centers $p$ using arc $p j l: j \in J, l \in L S_{p j}$

$T S_{j p l}$ : Time to transport any quantity of products between distribution centers $j$ using the arc $j p l ; j \in j, l \in L S_{j p}$

$T S_{p j l}$ : Time to transport any quantity of products between distribution centers $p$ using the arc $p j l ; j \in j, l \in L S_{p j}$.

\section{Decision Variables}

$R_{j p l}$ : Quantity transported from $j$ to $p$ using arc $j p l ; j \in J$, $l \in L S_{j p}$

$R_{p j l}$ : Quantity transported from $p$ to $j$ using arc $p j l ; j \in J, l$ $\in L S_{p j}$

$C_{j p l}$ : Binary variable equal to 1 if the arc $j p l$ is used to transport product between distribution centers $(j-p)$ and equals 0 otherwise; $j \in J, p \in P, l \in L S_{j p}$

$C_{p j l}$ : Binary variable equal to 1 if the arc $p j l$ is used to transport product between distribution centers $(p-j)$ and equals 0 otherwise; $j \in J, p \in P, l \in L S_{p j}$.

\section{Auxiliary Variables}

$T$ : Maximum time it takes to ship the product using the alternative $i, j, p, k, E_{3}=\max \left(T P_{i j l} A_{i j l}\right)+$ $\max \left(T S_{j p l} C_{j p l}\right)+\max \left(T W_{j k l} B_{j k l}\right) ; i \in I, j \in J, l \in$ $L P_{i j}, l \in L S_{j p}, k \in K, l \in L W_{j k}$

$E_{3}$ : Alternatively time $i-j-p-k, E_{3}=\underset{i j p k}{\operatorname{Max}}\left[T P_{i j l}+T S_{p j l}+\right.$ $\left.T W_{j k l}\right]$

$M$ : A very large positive value

$\delta_{j p}$ : Binary variable equal to 1 if the arc $j p l$ is used to transport product from $j$ to $p$ and equals 0 otherwise $j \in$ $J, l \in L S_{j p}$

$\delta_{p j}$ : Binary variable equal to 1 if the arc $p j l$ is used to transport product from $p$ to $j$ and equals 0 otherwise $\in J, l \in L S_{p j}$. 
Model 3. Consider $\min \left(f_{1}, f_{2}\right)$

$$
\begin{aligned}
& f_{1}= \sum_{i \in I} \sum_{j \in J} \sum_{l \in L P_{i j}} C P_{i j l} X_{i j l}+\sum_{j \in J} \sum_{k \in K} \sum_{l \in L W_{j k}} C W_{j k l} Y_{j k l} \\
&+\sum_{j \in J} \sum_{p \in P} \sum_{l \in L S_{j p}} C_{j p l} R_{j p l} \\
&+\sum_{j \in J} \sum_{p \in P} \sum_{l \in L S_{p j}} C_{p j l} R_{p j l}+\sum_{j \in J} F_{j} Z_{j} \\
&(2),(3),(4),(5),(6),(7),(8),(10), \\
&(11),(12),(13),(14),(15),(16), \\
& \sum_{l \in L S_{j p}} C_{j p l} \leq M\left(1-\delta_{j p}\right) \quad \forall j \in J, p \in P, \quad \forall j \in J, \\
& \sum_{l \in L S_{p j}} C_{p j l} \leq M\left(1-\delta_{p j}\right) \quad \forall j \in J, p \in P, \\
& \sum_{j \in J} \sum_{l \in L S_{j p}} C_{j p l}+\sum_{p \in P} \sum_{l \in L S_{j p}} C_{p j l} \leq 1 \quad \forall j \in J, p \in P, \\
& E_{3}-\sum_{l \in L P_{i j}} A_{i j l} T P_{i j l}+\sum_{l \in L S_{j p}} C_{j p l} T S_{j p l} \\
& \quad+\sum_{l \in L W_{p k}} B_{p k l} T W_{p k l} \geq-M \delta_{j p}
\end{aligned}
$$

$\forall i \in I, \quad j \in J, \quad p \in P, \quad k \in K$,

$$
\begin{array}{r}
E_{3}-\sum_{l \in L P_{i j}} A_{i p l} T P_{i p l}+\sum_{l \in L S_{p j}} C_{p j l} T S_{p j l} \\
+\sum_{l \in L W_{p k}} B_{j k l} T W_{j k l} \geq-M \delta_{p j}
\end{array}
$$

$\forall i \in I, \quad j \in J, \quad p \in P, \quad k \in K$,

$$
\begin{gathered}
M W_{j} Z_{j}-\left(\sum_{j \in J} \sum_{l \in L W_{j k}} Y_{j k l}+\sum_{j \in L S_{j p}} R_{j p l}-\sum_{j \in L S_{p j}} R_{p j l}\right) \geq 0 \\
\forall j \in J \\
\sum_{i \in I} \sum_{l \in L P_{i j}} X_{i j l}+\sum_{p \in P} \sum_{l \in L S_{j p}} R_{p j l} \\
-\sum_{k \in K} \sum_{l \in L W_{j k}} Y_{j k l}-\sum_{p \in P} \sum_{l \in L S_{j p}} R_{j p l}=0
\end{gathered}
$$

$$
\forall j \in J,
$$

$$
\begin{gathered}
\sum_{l \in L S_{j p}} C_{j p l} \leq 1 \quad \forall j \in J, \\
\sum_{l \in L S_{p j}} C_{p j l} \leq 1 \quad \forall j \in J, \\
\sum_{p \in P} \sum_{l \in L S_{j p}} C_{j p l}+\sum_{p \in P} \sum_{l \in L S_{j p}} C_{p j l} \leq 1 \quad \forall j \in J, \\
R_{j p l}-C_{j p l} \geq 0 \quad \forall j \in J, l \in L S_{j p}, \\
R_{p j l}-C_{p j l} \geq 0 \quad \forall j \in J, l \in L S_{p j}, \\
M W_{j} C_{j p l}-R_{j p l} \geq 0 \quad \forall j \in J, l \in L S_{j p}, \\
M W_{j} C_{p j l}-R_{p j l} \geq 0 \quad \forall j \in J, \quad l \in L S_{p j} \\
T, E_{j}^{1}, E_{j}^{2}, E_{3}, X_{i j l}, Y_{j k l}, R_{j p l} \geq 0 \\
\forall i \in I, \quad j \in J, \quad k \in K, \quad l \in L P_{i j}, \\
l \in L W_{j k}, \quad l \in L S_{j p}, \\
Z_{j}, A_{i j l}, B_{j k l}, C_{j p l}, \delta_{j p} \in\{0,1\} \\
\forall i \in I, \quad j \in J, \quad k \in K, \quad l \in L P_{i j}, \\
\quad l \in L W_{j k}, \quad l \in L S_{j p} .
\end{gathered}
$$

In this new formulation (30) replaces (1) in the original model as an objective function that looks for the best possible cost. Equation (31) is added to the original model and calculates the longest time considering the flow $(j)-(p)$. Equations (32) and (33) are added to the original set as a condition if-then to determine the longest time in the flow of product from $(i)$ to $(k)$, considering the flow between $(j)$ and $(p)$. Equation (34) is added to the original model and this limits multiple routing between distribution centers $(j)$. Equations (35) and (36) are added to the original model and complete the if-then condition to calculate the longest time in the flow of product from $(i)$ to $(k)$ considering the flow of $(j)-(p)$. Equation (37) replaces (8) in the original model and states that the amount transported from $(j)$ to $(k)$ does not exceed the capacity of distribution center $(j)$ by considering the flow between distribution centers $(j)-(p)$. Equation (38) replaces (9) in the original model and provides the flow balance in the distribution centers. Equations (39) and (40) are added to the original model and these provide that the transportation of the material can only be done through a single arc. Equation (41) is added to the original model and states that the exchange of product between $(j)$ and $(p)$ does not create a cycle. Equations (42) and (43) provide that an arc will be inactive if there is no flow through it. Equations (44) and (45) are added to the original model and establish that the distribution centers $(j)$, having no product flow through them, will be closed. Equations (46) and (47) replace (18) and (19) in the original model and set the domain of the variables in the model.

\section{Computational Experiment}

For the process of computational experiments, five sets of instances of each size were used as shown in Table 1. 
TABLE 1: Instances sizes.

\begin{tabular}{|c|c|c|c|c|}
\hline Instances sizes & $\begin{array}{l}\text { Integer variables in the } \\
\text { original model }\end{array}$ & $\begin{array}{l}\text { Integer variables in the } \\
\text { approach without single } \\
\text { source }\end{array}$ & $\begin{array}{l}\text { Integer variables in the } \\
\text { approach allowing direct } \\
\text { flow between }(i) \text { and }(k)\end{array}$ & $\begin{array}{l}\text { Integer variables in the } \\
\text { approach allowing flow } \\
\text { between distribution } \\
\text { centers }(j)-(p)\end{array}$ \\
\hline 5_5_5_2 & 105 & 105 & 205 & 355 \\
\hline 5_5_5_5 & 255 & 255 & 505 & 805 \\
\hline 5_10_10_2 & 320 & 320 & 510 & 1310 \\
\hline 5_10_15_2 & 410 & 410 & 710 & 1410 \\
\hline 5_10_20_2 & 510 & 510 & 910 & 1510 \\
\hline
\end{tabular}

The encoding of the instance sizes is as follows. The first index indicates the number of plants $(i)$, the second index indicates the number of distribution centers $(j)$, the third index indicates the number of customers $(k)$, and finally the fourth index indicates the number of arcs between nodes in each echelon. In each size, 5 instances were tested.

4.1. Instance Design. The instances used in the experimental process were initially proposed by $[2,3]$. These instances were used to test the original model and the variation that allows multiple sources of supply for customers.

For the variation that permits direct supply from the plants $(i)$ to customers $(k)$, these instances were extended to accommodate this approach. The times for the arcs of the alternative $(i)-(k)$ were generated at random on the basis of a normal distribution, with values ranging from 5 to 50 . The aim of these values is that they are competitive with those of the original instance so that the model can choose to select sometimes the direct flow of $(i)-(k)$ and in other cases to select the flow $(i)-(j)-(k)$. We consider that time and cost for the alternative $(i)-(k)$ are negatively correlated. The unit cost of transportation in the flow $(i)-(k)$ is a floating point variable calculated as follows:

$$
\text { Cost }_{1}=\frac{(7) *(50)}{\text { Time }} .
$$

This factor of " 7 " was determined after some experiments with some instances in such a way that the different solutions along the Pareto front included direct paths $(i)-(k)$ and indirect paths $(i)-(j)-(k)$. This was done to impede that the model would prefer a certain type of path.

To test the model that allows the exchange of product between distribution centers, we intended to use the original data extended by adding new times and costs for the arcs that allow the exchange between distribution centers $(j)$. With these data the model did not select $\operatorname{arcs}(j)-(p)$, because in fact the solutions had few open distribution centers $(j)$. To promote the opening of more distribution centers the fixed opening cost should be reduced in the new instances. With this change, we expected that some $\operatorname{arcs}(j)-(p)$ could be used in the solutions. Therefore, the extension to the original instance is as follows: the fixed cost of distribution center $(j)$ was generated as a random variable with integer values between 10,000 and 15,000.

These values will force the model to have more open distribution centers so that the product flow between them is more likely. From this point, the instances were expanded, creating additional time for flows $(j)-(p)$ at random with a range of 1 to 5 . The costs of the new alternatives are generated using the following relationship:

$$
\text { Cost }_{2}=\left(\frac{50}{\text { Time }}\right) *\left(\frac{1}{10}\right) .
$$

This factor of " $1 / 10$ " was determined after some experiments with some instances in such a way that the different solutions along the Pareto front included $(i)-(j)-(k)$ paths and $(i)-(j)-(p)-(k)$ paths. This was done to impede that the model would prefer a certain type of path.

To perform the computational experiment we used a computer equipped with the following features: Workstation with Intel (R) Xenon (R) CPU X5550 2.67 GHz with $12 \mathrm{~GB}$ of RAM and 64-bit operating system (Windows 7). The implementation of the models was performed in GAMS 12 and solved with CPLEX 23.6.2.

4.2. Metrics. To make the comparison of the new Pareto fronts with the original ones, the metric $R_{\text {pos }}\left(P_{i}\right)$ proposed by [4] was used. Additionally, we registered the average number of Pareto-optimal solutions in each front. To calculate the $R_{\text {pos }}\left(P_{i}\right)$, consider that $P_{1}$ and $P_{2}$ are the sets of Pareto-optimal solutions obtained from each model and $P$ is the union of the sets of Pareto-optimal solutions (i.e., $P=P_{1} \cup P_{2}$ ) such that it includes only nondominated solutions $Y$ 's. The ratio of Pareto-optimal solutions in $P_{i}$ that are not dominated by any other solutions in $P$ is calculated as follows:

$$
R_{\text {pos }}\left(P_{i}\right)=\frac{\left|P_{i}-\left\{X \in P_{i} \mid \exists T \in P: Y \prec X\right\}\right|}{P_{i}}
$$

where $Y \prec X$ means that the solution $X$ is dominated by solution $Y$. The higher the ratio $R_{\text {pos }}\left(P_{i}\right)$ is, the better the solution set $P_{i}$ is. Similarly, we used the metrics proposed by [3] called $D_{\text {avg }}$ and $D_{\min }$. These were developed to give practical meaning to the comparison of sets point by point. The discretization of objective $f_{2}$ and the number of objectives allow proceeding as follows for a pair of sets $S_{1}$ and $\mathrm{S}_{2}$.

Let $f_{1}$ and $f_{2}$ be the objective functions of the problem and $S_{1}$ and $S_{2}$ the sets of nondominated solutions to be compared. By discretization of function $f_{2}$ we can construct 
TABLE 2: Results of the first variation.

\begin{tabular}{|c|c|c|c|c|c|c|c|c|c|}
\hline \multirow{2}{*}{ Size } & \multicolumn{3}{|c|}{ With single source } & \multicolumn{3}{|c|}{ Without single source } & \multirow{2}{*}{$D_{\text {min }}$} & \multirow{2}{*}{$\begin{array}{c}\text { Time with } \\
\text { single source (seconds) }\end{array}$} & \multirow{2}{*}{$\begin{array}{l}\text { Time without } \\
\text { single source (seconds) }\end{array}$} \\
\hline & Instance & $\left|S_{i}\right|$ & $R_{\text {pos }}\left(S_{i}\right)$ & $\left|S_{i}\right|$ & $R_{\text {pos }}\left(S_{i}\right)$ & $D_{\text {avg }}$ & & & \\
\hline \multirow{5}{*}{ 5_5_5_2 } & 1 & 23 & 0.71875 & 32 & 1 & 0.99924159 & 0.98804728 & 8.838 & 9.068 \\
\hline & 2 & 13 & 0.46428571 & 28 & 1 & 0.99736445 & 0.98254824 & 9.197 & 11.052 \\
\hline & 3 & 15 & 0.53571429 & 28 & 1 & 0.99803935 & 0.99272805 & 9.606 & 10.751 \\
\hline & 4 & 18 & 0.5625 & 32 & 1 & 0.99726561 & 0.97547912 & 14.514 & 15.811 \\
\hline & 5 & 24 & 0.96 & 25 & 1 & 0.99932249 & 0.98894956 & 9.006 & 9.565 \\
\hline \multirow{5}{*}{ 5_5_5_5 } & 1 & 34 & 0.89473684 & 38 & 1 & 0.99955038 & 0.99126315 & 40.998 & 59.376 \\
\hline & 2 & 8 & 0.2 & 40 & 1 & 0.99470283 & 0.97594721 & 55.89 & 68.319 \\
\hline & 3 & 25 & 0.64102564 & 39 & 1 & 0.99933261 & 0.98922985 & 68.772 & 87.766 \\
\hline & 4 & 30 & 0.76923077 & 39 & 1 & 0.99938233 & 0.99327893 & 55.651 & 93.328 \\
\hline & 5 & 37 & 0.94871795 & 39 & 1 & 0.99993551 & 0.99766135 & 41.012 & 52.617 \\
\hline \multirow{5}{*}{ 5_10_10_2 } & 1 & 0 & 0 & 37 & 1 & 0.96917717 & 0.92948071 & 356.355 & 2294.165 \\
\hline & 2 & 0 & 0 & 39 & 1 & 0.98128174 & 0.96600743 & 193.005 & 371.465 \\
\hline & 3 & 0 & 0 & 37 & 1 & 0.98124332 & 0.96250708 & 417.547 & 2212.05 \\
\hline & 4 & 2 & 0.05 & 40 & 1 & 0.97790924 & 0.94280589 & 233.841 & 813.129 \\
\hline & 5 & 0 & 0 & 37 & 1 & 0.96969167 & 0.91712171 & 259.442 & 1674.431 \\
\hline \multirow{5}{*}{ 5_10_15_2 } & 1 & 0 & 0 & 36 & 1 & 0.97493663 & 0.93690112 & 8042.069 & 29027.322 \\
\hline & 2 & 0 & 0 & 36 & 1 & 0.98081129 & 0.95607975 & 11207.506 & 2668.63 \\
\hline & 3 & 0 & 0 & 35 & 1 & 0.98012085 & 0.96681874 & 16262.752 & 8218.611 \\
\hline & 4 & 0 & 0 & 37 & 1 & 0.98183982 & 0.96352299 & 23225.921 & 10991.189 \\
\hline & 5 & 0 & 0 & 39 & 1 & 0.98417036 & 0.96930275 & 8734.642 & 6810.192 \\
\hline \multirow{5}{*}{ 5_10_20_2 } & 1 & 0 & 0 & 35 & 1 & 0.98737854 & 0.97111859 & 149028.323 & 364.128 \\
\hline & 2 & 0 & 0 & 37 & 1 & 0.98613018 & 0.97454929 & 112315.372 & 24367.128 \\
\hline & 3 & 0 & 0 & 35 & 1 & 0.99016256 & 0.9824318 & 246814.212 & 38991.51 \\
\hline & 4 & 0 & 0 & 37 & 1 & 0.98522424 & 0.973581 & 240520 & 39919.572 \\
\hline & 5 & 0 & 0 & 37 & 1 & 0.98692727 & 0.97677506 & 78406 & 32163.298 \\
\hline
\end{tabular}

a set $T$, such that its elements are those values of $f_{2}$ that exist in $S_{1}$ and $S_{2}$,

$$
\begin{gathered}
T=\left\{f_{2}(s) \vee f_{2}\left(s^{\prime}\right), s \in S_{1}, s^{\prime} \in S_{2} \mid \exists f_{1}(s) \wedge \exists f_{1}\left(s^{\prime}\right)\right. \\
\left.\wedge f_{2}(s)=f_{2}\left(s^{\prime}\right)\right\} .
\end{gathered}
$$

Then $D_{\text {avg }}$ computes an average rate deviation of the objective function $f_{1}$ for each value of $f_{2}$, which is in the set T,

$$
\begin{gathered}
D_{\mathrm{avg}}=\frac{\sum_{t \in T}\left(\left(f_{1}(s): f_{2}(s)=t\right) /\left(f_{1}\left(s^{\prime}\right): f_{2}\left(s^{\prime}\right)=t\right)\right)}{|T|} \quad \forall s \in S_{1}, \quad s^{\prime} \in S_{2}, \\
D_{\min }=\min _{t \in T} \frac{\left(f_{1}(s): f_{2}(s)=t\right)}{\left(f_{1}\left(s^{\prime}\right): f_{2}\left(s^{\prime}\right)=t\right)} \\
\forall s \in S_{1}, \quad s^{\prime} \in S_{2} .
\end{gathered}
$$

The metric $D_{\text {avg }}$ indicates the quality of a set compared to another. The following relationship can be established:

$$
\text { If } D_{\text {avg }} \begin{cases}<1 & S_{1} \text { is better than } S_{2} \\ >1 & S_{1} \text { is worse than } S_{2} \\ =1 & S_{1} \text { is similar to } S_{2} .\end{cases}
$$

It is important to establish that an important parameter is the computational time required to solve each model for an instance.

4.3. Results. Table 2 shows the comparison results for the solution of the original model and the first variation (without single source constraint) of the CFCLP-TC problem. In this evaluation five instances are presented with metrics $R_{\text {pos }}$, $D_{\text {avg }}$, and $D_{\min }$ and the processing time in seconds of each one.

Table 2 shows that in all cases $\mathrm{D}_{\text {avg }}$ is less than 1 ; this indicates a superior quality of the Pareto fronts of the variation compared to the original model. $\mathrm{D}_{\min }$ in all cases indicates a small difference between both fronts, since the values are close to 1 . The values of $R_{\text {pos }}$ in the original model are on average $64 \%$ for instance $5-5-5-2,69 \%$ for instances 
TABLE 3: Results of the second variation.

\begin{tabular}{|c|c|c|c|c|c|c|c|c|c|}
\hline \multirow[t]{2}{*}{ Size } & \multirow[t]{2}{*}{ Instance } & \multicolumn{2}{|c|}{ Original model } & \multicolumn{2}{|c|}{$\begin{array}{c}\text { Direct flow between }(i) \\
\text { and }(k)\end{array}$} & \multirow[t]{2}{*}{$D_{\text {avg }}$} & \multirow[t]{2}{*}{$D_{\min }$} & \multirow[t]{2}{*}{$\begin{array}{l}\text { Time with original } \\
\text { model (seconds) }\end{array}$} & \multirow[t]{2}{*}{$\begin{array}{l}\text { Time direct flow between } \\
(i) \text { and }(k) \text { (seconds) }\end{array}$} \\
\hline & & $\left|S_{i}\right|$ & $R_{\text {pos }}\left(S_{i}\right)$ & $\left|S_{i}\right|$ & $R_{\mathrm{pos}}\left(S_{i}\right)$ & & & & \\
\hline \multirow{5}{*}{ 5_5_5_2 } & 1 & 27 & 0.87096774 & 31 & 1 & 0.9975774 & 0.95965946 & 8.838 & 9.263 \\
\hline & 2 & 15 & 0.42857143 & 35 & 1 & 0.98096094 & 0.91788812 & 9.197 & 10.276 \\
\hline & 3 & 18 & 0.54545455 & 33 & 1 & 0.96701778 & 0.85386306 & 9.606 & 10.456 \\
\hline & 4 & 1 & 0.03225806 & 31 & 1 & 0.95896192 & 0.91764374 & 14.514 & 12.989 \\
\hline & 5 & 23 & 0.79310345 & 29 & 1 & 0.99362365 & 0.96388563 & 9.006 & 9.018 \\
\hline \multirow{5}{*}{ 5_5_5_5 } & 1 & 38 & 0.92682927 & 41 & 1 & 1 & 1 & 40.977 & 72.21 \\
\hline & 2 & 33 & 0.76744186 & 43 & 1 & 0.99936964 & 0.99153492 & 55.504 & 99.961 \\
\hline & 3 & 16 & 0.38095238 & 42 & 1 & 0.98025659 & 0.93238372 & 68.331 & 106.332 \\
\hline & 4 & 35 & 0.81395349 & 43 & 1 & 0.99788515 & 0.97854988 & 55.618 & 105.304 \\
\hline & 5 & 34 & 0.79069767 & 43 & 1 & 0.99839478 & 0.96884197 & 40.962 & 69.114 \\
\hline \multirow{5}{*}{ 5_10_10_2 } & 1 & 18 & 0.46153846 & 39 & 1 & 0.98846976 & 0.94669285 & 351.812 & 528.167 \\
\hline & 2 & 32 & 0.7804878 & 41 & 1 & 0.99766759 & 0.97732813 & 192.369 & 293.994 \\
\hline & 3 & 27 & 0.675 & 40 & 1 & 0.99658614 & 0.96356955 & 416.336 & 540.134 \\
\hline & 4 & 31 & 0.775 & 40 & 1 & 0.99855269 & 0.98577745 & 233.123 & 393.202 \\
\hline & 5 & 19 & 0.52777778 & 36 & 1 & 0.98881179 & 0.89438732 & 259.084 & 341.4 \\
\hline \multirow{5}{*}{ 5_10_15_2 } & 1 & 2 & 0.555556 & 36 & 1 & 0.91196956 & 0.7597195 & 6803.334 & 444.791 \\
\hline & 2 & 11 & 0.28205128 & 36 & 1 & 0.98962601 & 0.94587785 & 9754.167 & 10448.886 \\
\hline & 3 & 11 & 0.275 & 39 & 1 & 0.97893561 & 0.86283473 & 9105.095 & 6400.084 \\
\hline & 4 & 14 & 0.35 & 40 & 1 & 0.97651925 & 0.87874916 & 12192.357 & 10856.243 \\
\hline & 5 & 18 & 0.15 & 40 & 1 & 0.98074666 & 0.91927882 & 7987.301 & 7619.696 \\
\hline \multirow{5}{*}{$5 \_10 \_20 \_2$} & 1 & 3 & 0.07692308 & 39 & 1 & 0.94659341 & 0.80255363 & 149028.323 & 137238.826 \\
\hline & 2 & 9 & 0.24324324 & 37 & 1 & 0.95456087 & 0.83359301 & 112315.372 & 112106.767 \\
\hline & 3 & 10 & 0.26315789 & 38 & 1 & 0.96070312 & 0.83766502 & 246814.212 & 129141.434 \\
\hline & 4 & 8 & 0.20512821 & 39 & 1 & 0.96055131 & 0.83801063 & 240520.165 & 246519.6 \\
\hline & 5 & 9 & 0.23076923 & 39 & 1 & 0.96889729 & 0.56548166 & 78406.285 & 467645.918 \\
\hline
\end{tabular}

5-5-5-5, and $0 \%$ for instances 5-10-10-2, 5-10-15-2, and 5-1020-2. This indicates that in all cases the variation that allows the supply of product to customers without single source constraint has always better Pareto fronts compared with those obtained in the original model.

The processing time for the variation increased on average by $9 \%$ for instances $5-5-5-2$, increased on average $627 \%$ for instances $5-5-5-5$, and increased by $76 \%$ on average for instances 5-10-10-2 compared to the original model. However for instances $5-10-15-2$ time decreased by $16 \%$ and by $500 \%$ for instances 5-10-20-2.

In Table 3 the results of the comparison between the original model and the variation that allows the direct flow between the plants $(i)$ and customers $(k)$ are shown. It should be noted that, in the majority of the cases, $D_{\text {avg }}$ is less than 1 ; this indicates that the Pareto fronts of the variation are better compared to the original model. $D_{\min }$ in all cases indicates the smallest difference comparing both fronts and provides a measure of the difference of the fronts compared.

In relation to $R_{\text {pos }}$ it is observed that the variation of the model presents values of 1 in all cases, compared with the original model. And the values of $R_{\text {pos }}$ in the original model are below the proposed variation on average $53 \%$ for instance $5-5-5-2,73 \%$ for instance $5-5-5-5,64 \%$ for the instances $5-10-10-2,26 \%$ for instance $5-10-15-2$, and $18 \%$ for instance 5-10-20-2. This indicates that in all cases the direct flow variation between $(i)$ and $(k)$ has always better Pareto fronts as compared with those obtained in the original model.

Concerning the processing time, comparing the original model with the variation that allows the flow from $(i)$ to $(k)$, we have the following: for instance 5-5-5-2 time is increased on average by $1.6 \%$ and for instance $5-5-5-5$ increased on average by $6.2 \%$ over the original model, for instance 5-10-102 a decrease is observed on average by $3.4 \%$, and for instances $5-10-15-2$ and $5-10-20-2$ processing time decreased by $22 \%$ and increased by $16 \%$, respectively.

Table 4 shows the results of the variation that allows the flow between distribution centers $(j)-(p) . D_{\text {avg }}$ is greater than 1 in all cases; this indicates a lower quality of the Pareto fronts of this variation compared with the original model.

The $R_{\text {pos }}$ values in the original model are on average of 1 with respect to the variation; this indicates that the original model presents always better Pareto fronts. With respect to processing time, for instance 5-5-5-2 time is increased by 
TABLE 4: Results of the third variation.

\begin{tabular}{|c|c|c|c|c|c|c|c|c|c|}
\hline \multirow[t]{2}{*}{ Size } & \multirow{2}{*}{ Instance } & \multicolumn{2}{|c|}{ Original model } & \multicolumn{2}{|c|}{$\begin{array}{c}\text { Direct flow between }(j) \\
\text { and }(p)\end{array}$} & \multirow[t]{2}{*}{$D_{\text {avg }}$} & \multirow[t]{2}{*}{$D_{\min }$} & \multirow{2}{*}{$\begin{array}{l}\text { Time with original } \\
\text { model (seconds) }\end{array}$} & \multirow{2}{*}{$\begin{array}{c}\text { Time flow between }(j) \\
\text { and }(p) \text { (seconds) }\end{array}$} \\
\hline & & $\left|S_{i}\right|$ & $R_{\text {pos }}\left(S_{i}\right)$ & $\left|S_{i}\right|$ & $R_{\text {pos }}\left(S_{i}\right)$ & & & & \\
\hline \multirow{5}{*}{$5 \_5 \_5 \_2$} & 1 & 33 & 1 & 11 & 0.33333333 & 1.0165715 & 1 & 11.631 & 326.195 \\
\hline & 2 & 31 & 1 & 1 & 0.03225806 & 1.01402981 & 1 & 20.896 & 292.527 \\
\hline & 3 & 31 & 1 & 0 & 0 & 1.04683427 & 1.02795867 & 8.728 & 245.921 \\
\hline & 4 & 32 & 1 & 0 & 0 & 1.06735052 & 1.006421 & 13.499 & 1215.217 \\
\hline & 5 & 30 & 1 & 0 & 0 & 1.08324319 & 1.03670392 & 9.841 & 898.683 \\
\hline \multirow{5}{*}{ 5_5_5_5 } & 1 & 38 & 1 & 0 & 0 & 1.02172283 & 1.00771899 & 187.27 & 52861.828 \\
\hline & 2 & 39 & 1 & 0 & 0 & 1.01398614 & 1.00201322 & 112.811 & 382637.904 \\
\hline & 3 & 39 & 1 & 6 & 0.15384615 & 1.00778561 & 1 & 3944.193 & 122663.847 \\
\hline & 4 & 40 & 1 & 0 & 0 & 1.01458474 & 1.00033388 & 218.698 & 43666.763 \\
\hline & 5 & 40 & 1 & 2 & 0.05 & 1.01185138 & 1 & 2234.115 & 131359.178 \\
\hline
\end{tabular}

average of $460 \%$. For instance 5-5-5-5 time is increased on average by $560 \%$ over the original model.

For the analysis of this variation only the first two groups of instances were solved, because the processing time required for evaluating the variation was very long. Solving some instances of the group 5-5-5-5 required up to 34 hours to get results, however for instances 5-10-10-2 after 143 hours of processing the solver did not yield a result, so we decided not testing for the rest of the instances.

\section{Conclusions}

Reference [1] defines the management of the supply chain as the process of planning, implementation, and operational control of the supply chain in an efficient manner. This aspect is defined in the context of tactical decisions that allow for more efficiency in the full cycle of manufacture. The work developed in this research explores an area that has not been sufficiently analyzed and incorporated into mathematical models of supply chain design with selection of distribution channels according to $[40,41]$.

The CFCLP-TC proposed by [2,3] incorporates in a novel way the selection of transportation alternatives in the context of a (two-echelon) problem considering plants, distribution centers, and customers. However, the changes proposed in this paper can bring theoretical models to real applications, as they consider situations that could occur in real context.

The first variation that allows the flow to customers from multiple sources (DC) states that the proposed approach generally results in better cost compared with the original proposal where customers are limited to obtaining the product from a single source (DC). This enables better Pareto fronts in the proposed alternative. Although the results obtained suggest that it is cheaper to allow customers to be supplied by more than a distribution center, this increases the complexity of cross-docking, and in accordance with [40] the increase of the level of complexity of the supply chain will negatively affect the performance of a manufacturing plant. However, a greater variety of products may create economies of a scale enough to reduce this effect.
The second variation allows in some cases the product flows from plants directly to customers without necessarily passing through the distribution centers. This variation generally obtained better costs compared to the original proposal. The proposal can be justified when customers are geographically closer to the plants than the distribution centers.

When planning the configuration of the supply chain, it is not possible to accurately predict in the future where they will reside geographically, and it is clear that it is more costly to relocate distribution centers based on the geographical configuration of new customers. Therefore, an alternative that would optimize the cost and time of the supply chain is based on the ability to send directly from plants to customers. The results obtained allowed us to determine the best Pareto fronts with this approach; however, a problem is the time required to solve the instances, since the increase of time is significant as we try to solve the larger instances that resembled real problems. The proposal explores a configuration of the new supply chain that has not been considered in the literature.

The third variation allowing in some cases the exchange of product between distribution centers generally gets worse costs compared with the original model. This proposal is considered in the overall context of managing the supply chain, where a distribution center can supply to another distribution center if the times to get the product to the customer and the costs associated with this alternative are lower. An example is the exchange of product between car dealers to reduce lead times, since placing an order to assembly plant takes time and cost, and these impact the service level.

The results determined that this approach provides Pareto fronts worse than the original model and therefore they have higher costs. For this approach, processing times are greatly increased as the size of the instances grows; this situation is not rare in practice. But one aspect that the model does not consider is the level of customer service, since for the example above it is difficult to predict with accuracy the products requested by the customers, and when this happens, you must 
deliver the product the customer demands in the shortest possible time and cost.

The proposed approaches are novel and allow mathematical modeling to configure supply chains to situations that occur most commonly in practice and allow closing the gap between theory and practice, contributing to the state of the art in this context.

It is clear that the new approaches produced an increase in the required processing time. The findings and conclusions presented are based only on instances that were tested, which in a context of real implementation are of small size. It is possible that the results of the Pareto front for larger instances may be different; therefore, it is important to determine these fronts, and however the impossibility of doing so with exact methods requires us to try to get them with heuristics and metaheuristics. In the revised literature the Lagrangian relaxation method is widely used to solve similar problems [42], and this may be applied to the problem addressed in this work. Another approach is the use of genetic algorithms proposed by [43] for a similar problem found in the literature. Evolutionary algorithms like Nondominated Sorting Genetic Algorithm (NSGA-II) and Strength Pareto Evolutionary Algorithm (SPEA-II) are widely used in multiobjective problems as in $[17,44,45]$.

\section{Conflict of Interests}

The authors declare that there is no conflict of interests regarding the publication of this paper.

\section{Acknowledgments}

The authors appreciate the support of the administration of Polytechnic University of Tulancingo, for the equipment and facilities provided for the development of this work, as well as the comments from the Faculty of Applied Mathematics and Approximation in the same university.

\section{References}

[1] M. T. Melo, S. Nickel, and F. Saldanha-da-Gama, "Facility location and supply chain management-a review," European Journal of Operational Research, vol. 196, no. 2, pp. 401-412, 2009.

[2] E. Olivares Benitez, Capacitated fixed cost facility location problem with transportation choices [Ph.D. thesis], ITESM, 2007.

[3] E. Olivares-Benitez, J. L. González-Velarde, and R. Z. RíosMercado, "A supply chain design problem with facility location and bi-objective transportation choices," TOP, vol. 20, no. 3, pp. 729-753, 2012.

[4] F. Altiparmak, M. Gen, L. Lin, and T. Paksoy, "A genetic algorithm approach for multi-objective optimization of supply chain networks," Computers \& Industrial Engineering, vol. 51, no. 1, pp. 196-215, 2006.

[5] A. M. Geoffrion and R. F. Powers, "Twenty years of strategic distribution system design: an evolutionary perspective," Interfaces, vol. 25, no. 5, pp. 105-127, 1995.
[6] C. H. Aikens, "Facility location models for distribution planning," European Journal of Operational Research, vol. 22, no. 3, pp. 263-279, 1985.

[7] C. S. Revelle and G. Laporte, "The plant location problem: new models and research prospects," Operations Research, vol. 44, no. 6, pp. 864-874, 1996.

[8] J. A. Díaz and E. Fernández, "Hybrid scatter search and path relinking for the capacitated $p$-median problem," European Journal of Operational Research, vol. 169, no. 2, pp. 570-585, 2006.

[9] N. Mladenović, J. Brimberg, P. Hansen, and J. A. Moreno-Pérez, "The $p$-median problem: a survey of metaheuristic approaches," European Journal of Operational Research, vol. 179, no. 3, pp. 927-939, 2007.

[10] M. Baïou and F. Barahona, "On the $p$-median polytope of $Y$-free graphs," Discrete Optimization, vol. 5, no. 2, pp. 205-219, 2008.

[11] B. Goldengorin and D. Krushinsky, "Complexity evaluation of benchmark instances for the $p$-median problem," Mathematical and Computer Modelling, vol. 53, no. 9-10, pp. 1719-1736, 2011.

[12] M. Sun, "Solving the uncapacitated facility location problem using tabu search," Computers \& Operations Research, vol. 33, no. 9, pp. 2563-2589, 2006.

[13] I. Averbakh, O. Berman, Z. Drezner, and G. O. Wesolowsky, "The uncapacitated facility location problem with demanddependent setup and service costs and customer-choice allocation," European Journal of Operational Research, vol. 179, no. 3, pp. 956-967, 2007.

[14] H.-C. Huang and R. Li, "A $k$-product uncapacitated facility location problem," European Journal of Operational Research, vol. 185, no. 2, pp. 552-562, 2008.

[15] A. Klose and A. Drexl, "Facility location models for distribution system design," European Journal of Operational Research, vol. 162, no. 1, pp. 4-29, 2005.

[16] C.-H. Chen and C.-J. Ting, "Combining Lagrangian heuristic and ant colony system to solve the single source capacitated facility location problem," Transportation Research E, vol. 44, no. 6, pp. 1099-1122, 2008.

[17] L. Lin, M. Gen, and X. Wang, "Integrated multistage logistics network design by using hybrid evolutionary algorithm," Computers \& Industrial Engineering, vol. 56, no. 3, pp. 854-873, 2009.

[18] Z. Yao, L. H. Lee, W. Jaruphongsa, V. Tan, and C. F. Hui, "Multisource facility location-allocation and inventory problem," European Journal of Operational Research, vol. 207, no. 2, pp. 750-762, 2010.

[19] İ. K. Altınel, E. Durmaz, N. Aras, and K. C. Özkısacık, "A location-allocation heuristic for the capacitated multi-facility Weber problem with probabilistic customer locations," European Journal of Operational Research, vol. 198, no. 3, pp. 790799, 2009.

[20] K. E. Caggiano, P. L. Jackson, J. A. Muckstadt, and J. A. Rappold, "Efficient computation of time-based customer service levels in a multi-item, multi-echelon supply chain: a practical approach for inventory optimization," European Journal of Operational Research, vol. 199, no. 3, pp. 744-749, 2009.

[21] A. Klose and S. Görtz, "A branch-and-price algorithm for the capacitated facility location problem," European Journal of Operational Research, vol. 179, no. 3, pp. 1109-1125, 2007.

[22] A. A. Kuehn and M. J. Hamburger, "A heuristic program for locating warehouses," Management Science, vol. 9, no. 4, pp. 643-666, 1963. 
[23] B. M. Khumawala, "An efficient heuristic procedure for the capacitated warehouse location problem," Naval Research Logistics Quarterly, vol. 21, no. 4, pp. 609-623, 1974.

[24] M. R. Korupolu, C. G. Plaxton, and R. Rajaraman, "Analysis of a local search heuristic for facility location problems," in Proceedings of the 9th Annual ACM-SIAM Symposium on Discrete Algorithms (SODA '98), pp. 1-10, ACM, Philadelphia, Pa, USA, 1998.

[25] E. Rolland, D. A. Schilling, and J. R. Current, "An efficient tabu search procedure for the $p$-median problem," European Journal of Operational Research, vol. 96, no. 2, pp. 329-342, 1997.

[26] H. Delmaire, J. A. Díaz, E. Fernández, and M. Ortega, "Reactive GRASP and tabu search based heuristics for the single source capacitated plant location problem," INFOR Journal, vol. 37, no. 3, pp. 194-225, 1999.

[27] T. L. Magnanti and R. T. Wong, "Accelerating benders decomposition: algorithmic enhancement and model selection criteria," Operations Research, vol. 29, no. 3, pp. 464-484, 1981.

[28] J. M. Y. Leung and T. L. Magnanti, "Valid inequalities and facets of the capacitated plant location problem," Mathematical Programming, vol. 44, no. 1-3, pp. 271-291, 1989.

[29] K. Aardal, "Capacitated facility location: separation algorithms and computational experience," Mathematical Programming, vol. 81, no. 2, pp. 149-175, 1998.

[30] G. Laporte, F. V. Louveaux, and L. van Hamme, "Exact solution of a stochastic location problem by an integer L-shaped algorithm," Transportation Science, vol. 28, no. 2, pp. 95-103, 1994.

[31] S. Tragantalerngsak, J. Holt, and M. Rönnqvist, "Lagrangian heuristics for the two-echelon, single-source, capacitated facility location problem," European Journal of Operational Research, vol. 102, no. 3, pp. 611-625, 1997.

[32] L. A. Moncayo-Martínez and Z. Z. David, "Optimising safety stock placement and lead time in a assembly supply chain using bi-objetive MAX-MIN ant system," International Journal of Production Economics, vol. 145, no. 1, pp. 18-28, 2013.

[33] W.-C. Yeh and M.-C. Chuang, "Using multi-objective genetic algorithm for partner selection in green supply chain problems," Expert Systems with Applications, vol. 38, no. 4, pp. 4244-4253, 2011.

[34] R. Rajesh, S. Pugazhendhi, and K. Ganesh, "Simulated annealing algorithm for balanced allocation problem," The International Journal of Advanced Manufacturing Technology, vol. 61, no. 5-8, pp. 431-440, 2011.

[35] G. C. Cabrera Guillermo, E. Cabrera, R. Soto, L. J. M. Rubio, B. Crawford, and F. Paredes, "A hybrid approach using an artificial bee algorithm with mixed integer programming applied to a large-scale capacitated facility location problem," Mathematical Problems in Engineering, vol. 2012, Article ID 954249, 14 pages, 2012.

[36] Y.-S. Myung, H.-G. Kim, and D.-W. Tcha, "A bi-objective uncapacitated facility location problem," European Journal of Operational Research, vol. 100, no. 3, pp. 608-616, 1997.

[37] J. G. Villegas, F. Palacios, and A. L. Medaglia, "Solution methods for the bi-objective (cost-coverage) unconstrained facility location problem with an illustrative example," Annals of Operations Research, vol. 147, no. 1, pp. 109-141, 2006.

[38] R. D. Galvão, L. G. A. Espejo, B. Boffey, and D. Yates, "Load balancing and capacity constraints in a hierarchical location model," European Journal of Operational Research, vol. 172, no. 2, pp. 631-646, 2006.
[39] M. da Graça Costa, M. E. Captivo, and J. Clímaco, "Capacitated single allocation hub location problem-a bi-criteria approach," Computers \& Operations Research, vol. 35, no. 11, pp. 3671-3695, 2008.

[40] C. C. Bozarth, D. P. Warsing, B. B. Flynn, and E. J. Flynn, "The impact of supply chain complexity on manufacturing plant performance," Journal of Operations Management, vol. 27, no. 1, pp. 78-93, 2009.

[41] J. Mula, D. Peidro, M. Díaz-Madroñero, and E. Vicens, "Mathematical programming models for supply chain production and transport planning," European Journal of Operational Research, vol. 204, no. 3, pp. 377-390, 2010.

[42] H. Lidestam and M. Rönnqvist, "Use of Lagrangian decomposition in supply chain planning," Mathematical and Computer Modelling, vol. 54, no. 9-10, pp. 2428-2442, 2011.

[43] M. Gen, F. Altiparmak, and L. Lin, "A genetic algorithm for twostage transportation problem using priority-based encoding," OR Spectrum, vol. 28, no. 3, pp. 337-354, 2006.

[44] X. Li, L. Amodeo, F. Yaloui, and H. Chehade, "A multiobjetive optimization approach to solve parallel machines scheduling problem," Advances in Artificial Intelligence, vol. 2010, Article ID 943050, 10 pages, 2010.

[45] H. Xing and R. Qu, "A nondominated sorting genetic algorithm for bi-objetive network coding based multicast routing problems," Information Sciences, vol. 233, pp. 36-53, 2013. 


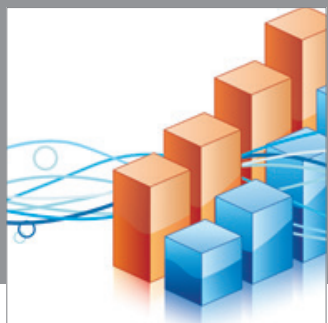

Advances in

Operations Research

mansans

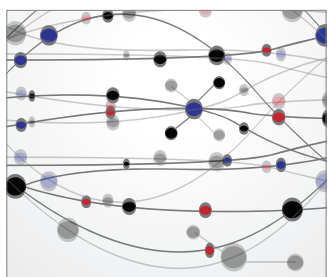

The Scientific World Journal
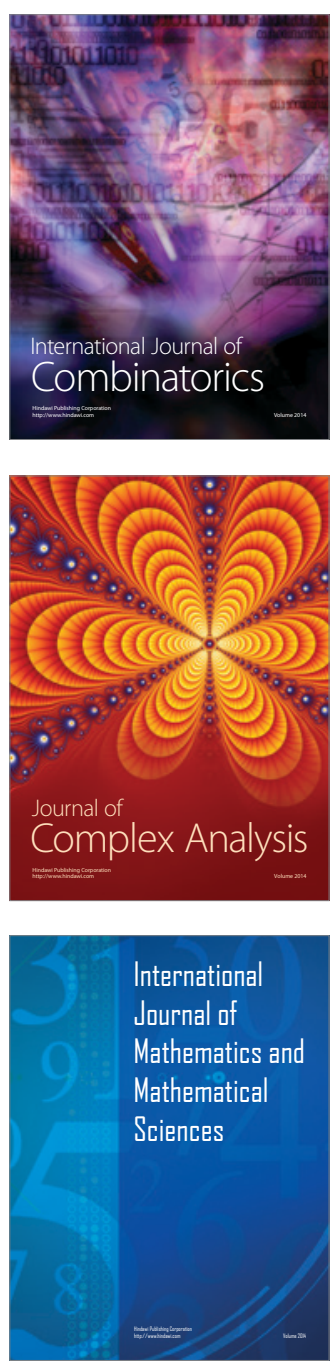
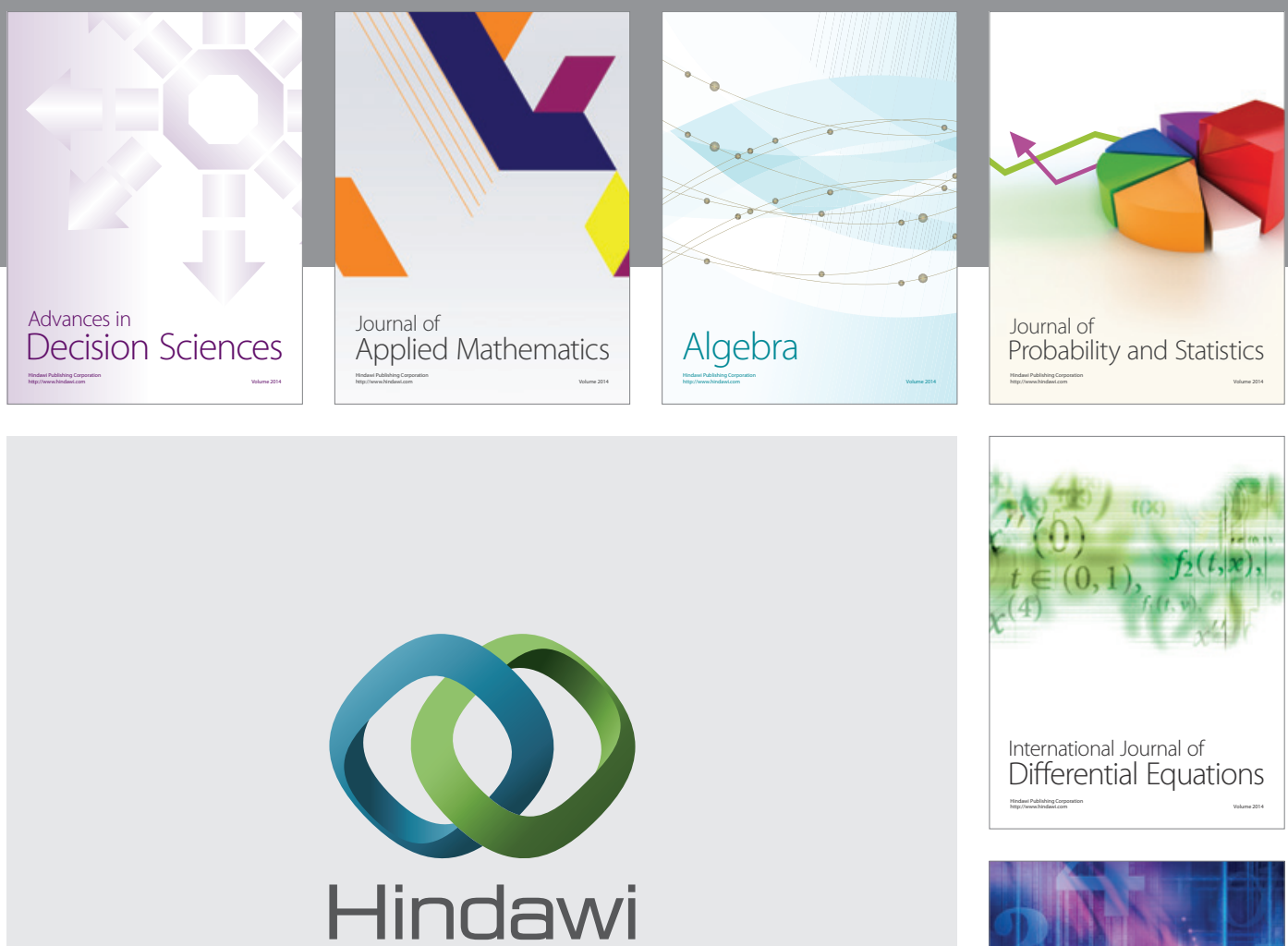

Submit your manuscripts at http://www.hindawi.com
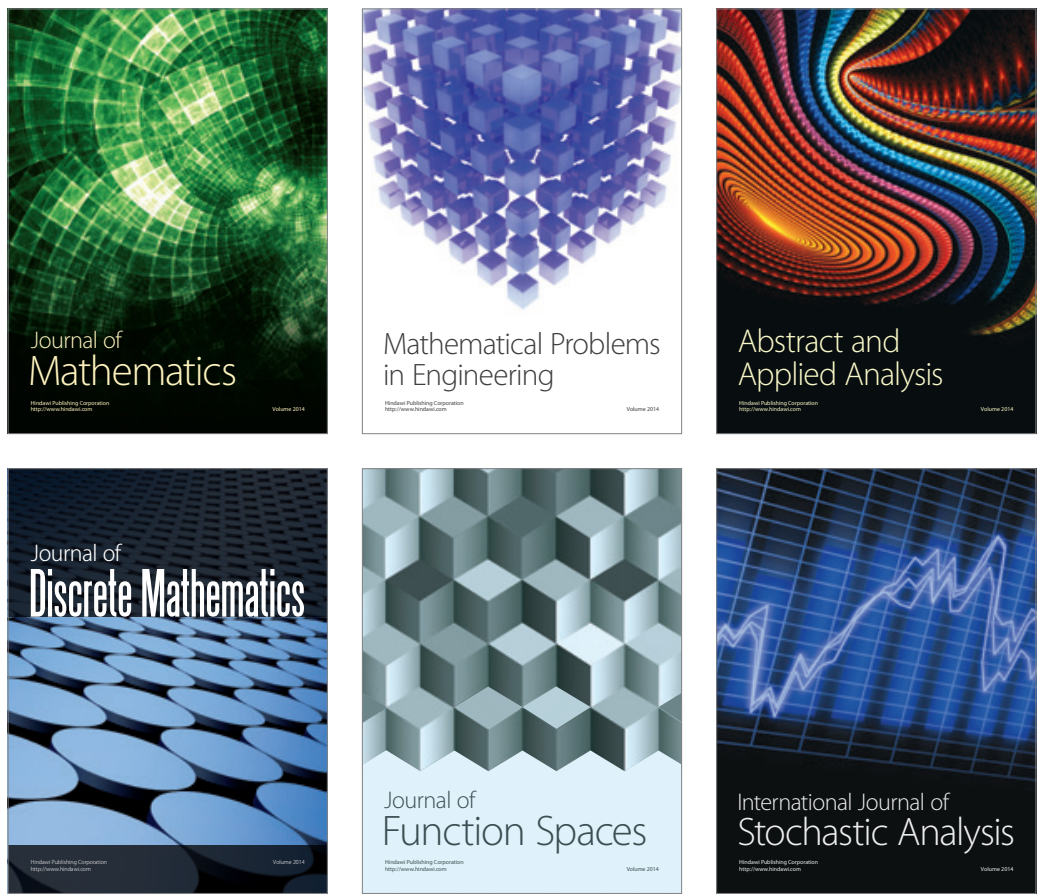

Journal of

Function Spaces

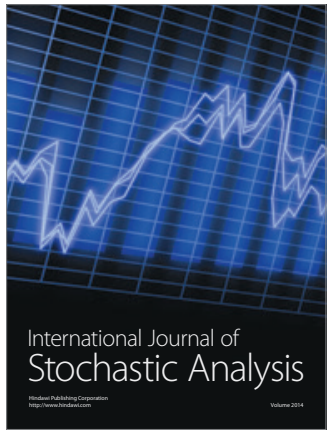

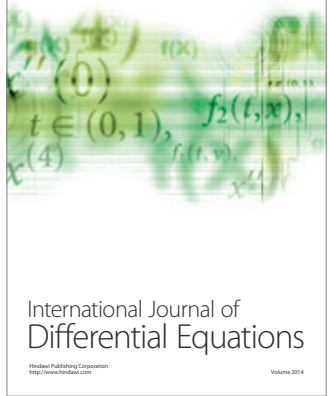
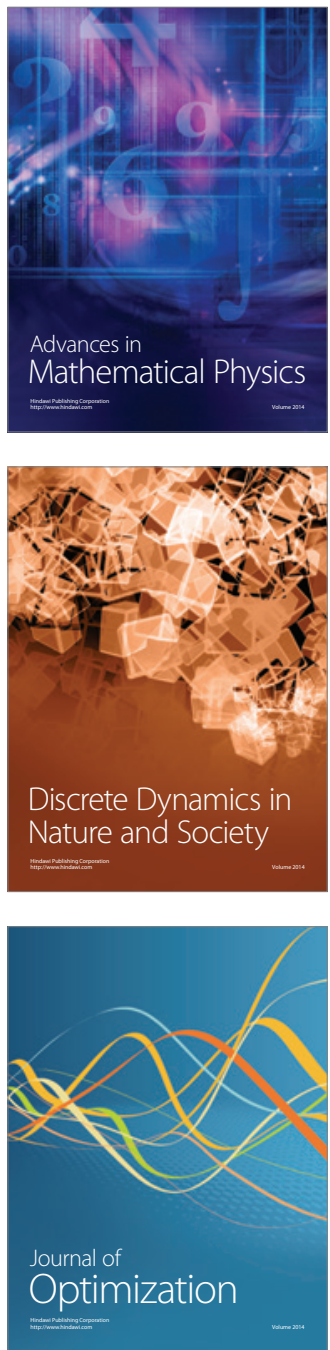